\title{
CINÉTICA DE CRECIMIENTO DE NANOPARTÍCULAS DE CdS CONFORMANDO MICROESTRUCTURAS LAS CUALES FUERON DEPOSITADAS POR PVD SOBRE SUSTRATOS METÁLICOS
}

\author{
THELMASERRANO, IDALIA GÓMEZ. \\ Laboratorio de Materiales I, Centro de Laboratorios Especializados, Facultad de Ciencias Químicas, \\ Universidad Autónoma de Nuevo León.
}

\begin{abstract}
Resumen. En este trabajo se presentan los resultados del estudio cinético de crecimiento de nanopartículas de CdS obtenidas vía microondas. Éstas fueron posteriormente depositadas sobre sustratos metálicos. Para la preparación de las nanopartículas se utilizaron soluciones de TAA, $\mathrm{CdCl}_{2}$ y $\mathrm{KOH}$ ajustando el $\mathrm{pH}$ a 8 , estas soluciones se irradiaron en un sistema de microondas a 1320W por un tiempo de un minuto. La deposición de las nanopartículas sobre sustratos metálicos se llevó a cabo en un sistema de deposición física, para lo cual se utilizó un horno tubular a una temperatura de $900^{\circ} \mathrm{C}$ con una rapidez de calentamiento de $10^{\circ} \mathrm{C} / \mathrm{min}$ y un tiempo de residencia de 2 horas, todo en atmósfera de Ar. Los sustratos metálicos utilizados fueron de acero al silicio y al manganeso. El estudio cinético se realizó mediante UV-Vis, encontrándose un crecimiento en tres etapas, obedeciendo a una ecuación tipo (1-n)(In-Inr)-In(n-1)=InK+ Int, donde la r es el radio de las nanopartículas, t es el tiempo la k la n la otra y la otra. Los depósitos mostraron una influencia de la morfología desarrollada en función del tipo de sustrato utilizado, obteniendo películas delgadas en los aceros al silicio, y morfologías tipo aguja en los aceros al manganeso.
\end{abstract}

\section{Introducción}

Las propiedades físicas y químicas de las nanopartículas son diferentes a las de los materiales bulk, originadas por los cambios en el espaciamiento de los niveles de energía y la alta relación área/volumen cuando el tamaño de la partícula disminuye. Un punto cuántico [1] es una partícula que tiene sus 3 dimensiones confinadas. Las propiedades ópticas [2,3] de puntos cuánticos tienen aplicaciones potenciales en optoelectrónica [4], dispositivos de memoria y fuentes de luz láser. Debido a la restricción espacial del movimiento del electrón y el hueco [5], la absorción óptica de nanopartículas semiconductoras se desplaza hacia energías mayores, es decir que el valor de la energía de banda prohibida $\mathrm{E}_{\mathrm{g}}$ [6], aumenta cuando el tamaño de la partícula decrece. Los puntos cuánticos se pueden sintetizar como dispersiones coloidales, donde los precursores del material están reaccionando en presencia de un agente estabilizador que restringe el crecimiento de la partícula, manteniéndola dentro de los límites del confinamiento cuántico[4].

En estos últimos años la ciencia ha dado un giro radical y ha puesto su interés en los semiconductores II-VI, más específicamente sulfuro de cadmio (CdS), ya que estos semiconductores tienen aplicación sensorial, óptica, solar y fotocatalítica. El CdS es un semiconductor que puede presentar dos arreglos cristalinos, uno de ellos es una estructura cúbica (blenda de zinc) y la otra hexagonal (wurtzita). El buen funcionamiento de un semiconductor está ligado a propiedades importantes como su cristalinidad y el tamaño de las partículas [7].

Las películas delgadas de CdS tienen muchas aplicaciones, por ejemplo en los aparatos optoeléctricos, ya que la conductividad del material se ve altamente favorecida en tal forma [8]. Para obtener este tipo de películas es necesario utilizar técnicas de deposición al alto vacío como evaporación, sputtering, entre otras, las cuales resultan inaccesibles y en ocasiones no viables debido a las condiciones de trabajo necesarias para su aplicación.

El objetivo planteado en este artículo es el de sintetizar nanopartículas de CdS y estudiar su estabilidad al modificar las concentraciones de citrato de sodio en el medio, además de realizar la deposición de las nanoestructuras sobre la superficie de diferentes tipos de aceros, [8-9] gracias a la afinidad existente entre dicho material y el semiconductor.

\section{Diseño experimental}

Se utilizó cloruro de cadmio anhidro ( $\mathrm{CdCl}_{2}$, Q.P. DEQ] como precursor del catión, Tioacetoamida $\left(\mathrm{CH}_{3} \mathrm{CSNH}_{2}\right)$ [MERCK 99\%] como precursor del anión, Citrato de sodio $\left(\mathrm{C}_{6} \mathrm{H}_{5} \mathrm{O}_{7} \cdot 2 \mathrm{H}_{2} \mathrm{O}\right)$ [DEQ 99\%] como estabilizador, $\mathrm{KOH}$ (grado reactivo, CTR Scientific) y agua destilada. Después se utilizó metanol $\left[\mathrm{CH}_{3} \mathrm{OH}\right.$ ] (absoluto 99.9\%, CTR Scientific) como agente de extracción y lavado de las nanopartículas; acetona $\left[\left(\mathrm{CH}_{3}\right)_{2} \mathrm{CO}\right]$ (grado reactivo, CTR Scientific) como agente dispersante, agua 
desionizada, etanol $\left[\mathrm{CH}_{3} \mathrm{CH}_{2} \mathrm{OH}\right]$ (absoluto $99.9 \%$, CTR Scientific), ácido nítrico concentrado (15 M) $\left[\mathrm{HNO}_{3}\right]$ (CTR Scientific) como agente de lavado de la superficie de los sustratos y argón [Ar] (grado cromatográfico, 99.998\% INFRA) para tener una atmósfera inerte durante la termodeposición.

Las dispersiones obtenidas se caracterizaron por UV-Vis en un espectrofotómetro Perkin Elmer lambda 12. Un análisis por DRX fue efectuado utilizando un difractómetro Siemens D5000 utilizando radiación Cu Ka $(\lambda=1.5418 \AA)$.Para el estudio se utilizó una lámpara de UV Spectroline con una longitud de onda de $365 \mathrm{~nm}$. Para el pulido de las placas se utilizó una pulidora Struers modelo LaboPol-1. Para la termodeposición del sulfuro de cadmio (II) en los sustratos metálicos se utilizó un Horno Tubular [HT] Barnstead/Thermolyne modelo 21100 utilizando un tubo de alta alúmina. Para la microscopía óptica (MO) se utilizó un microscopio óptico OLYMPUS modelo BX60 acoplado a un software de imagen Image Pro Plus 6.0

\subsection{Síntesis}

Se preparo una solución de $\mathrm{CdCl}_{2} 30 \mathrm{mM}$ y una segunda solución con de $\mathrm{CH}_{3} \mathrm{CSNH}_{2} 30 \mathrm{mM}$. Se ajusto el pH a 8 con KOH 0.012M. Posteriormente se mezclaron las tres soluciones y se sometieron a un tratamiento térmico en un horno de microondas de $2.45 \mathrm{GHz} 1650 \mathrm{~W}$ a una potencia del $80 \%$, por un minuto. Con las dispersiones obtenidas se realizó el estudio cinético, y para la deposición se precipitaron las partículas con una mezcla metanol: acetona 2:1, se lavaron los polvos con metanol y se centrifugaron. Se dejaron secar a temperatura ambiente por 24 horas y se obtuvieron los polvos de CdS.

\subsection{Estudio cinético.}

Se realizaron dos series de experimentos, en los cuales se modificaron las concentraciones del estabilizante las cuales variaron en valores de 1.70, $0.80,0.37 \mathrm{mM}$ las cuales fueron las concentraciones en la mezcla de reacción.

Las dispersiones obtenidas, se colocaron en dos sistemas. El sistema uno, en el cual se mantuvo presente una radiación UV de longitud de onda de $365 \mathrm{~nm}$, a una temperatura de $86^{\circ} \mathrm{C}$. El sistema dos, fue sometido a la radiación normal y temperatura ambiente. Los experimentos fueron monitoreados utilizando la técnica de UV-Vis y los espectros obtenidos sirvieron para obtener los cambios del tamaño con respecto al tiempo.

\subsection{Deposición del CdS en los Sustratos Metálicos}

Para efectuar la deposición en los sustratos metálicos estos fueron lavados con agua desionizada para eliminar impurezas de la superficie, posteriormente fueron pulidos hasta un acabado espejo y sometidos a un ataque químico con ácido nítrico alcohólico al $3 \% \mathrm{v} / \mathrm{v}$. Para la deposición en sustratos se utilizaron dos diferentes tipos de aceros como muestra la Tabla 1.

Tabla 1. Composición de los Sustratos Utilizados para la deposición de CdS

\begin{tabular}{|c|c|}
\hline Acero 1 & Acero 2 \\
\hline $\mathrm{Mn}(0.25 \%)$ & $\mathrm{Si}(0.45 \%)$ \\
\hline $\mathrm{C}(0.10 \%)$ & $\mathrm{C}(0.04 \%)$ \\
\hline $\mathrm{Fe}(99.65 \%)$ & $\mathrm{Fe}(99.51 \%)$ \\
\hline
\end{tabular}

Se colocó el CdS en un crisol y se introdujo dentro del tubo de alta alúmina, el sustrato se colocó sobre el crisol y se programó el HT para que se calentara desde la temperatura ambiente $\left(20^{\circ} \mathrm{C}\right)$ hasta $900{ }^{\circ} \mathrm{C}$ con una rampa de calentamiento de $10^{\circ} \mathrm{C} / \mathrm{min}$, con un tiempo de residencia de 2 horas y su posterior enfriamiento hasta temperatura ambiente, siempre en una atmósfera de Ar. Finalmente se retiró el sustrato metálico del tubo y se guardó en un desecador con atmósfera de Ar para su posterior caracterización.

\section{Resultados y discusión}

Las diferencias en longitudes de onda de absorción en el espectro UV-Vis del CdS se deben a un incremento en el tamaño de las partículas presentes en el sistema. ${ }^{2-3}$ Lo anterior se observa claramente en la Fig. 1, donde las partículas de tamaño superior $(\approx 10 \mathrm{~nm})$ empiezan a absorber a longitudes de onda superiores a $450 \mathrm{~nm}$, mientras que las partículas más pequeñas alrededor de $(\approx 3 \mathrm{~nm})$, absorben a longitudes de onda alrededor de 420-430 nm. 


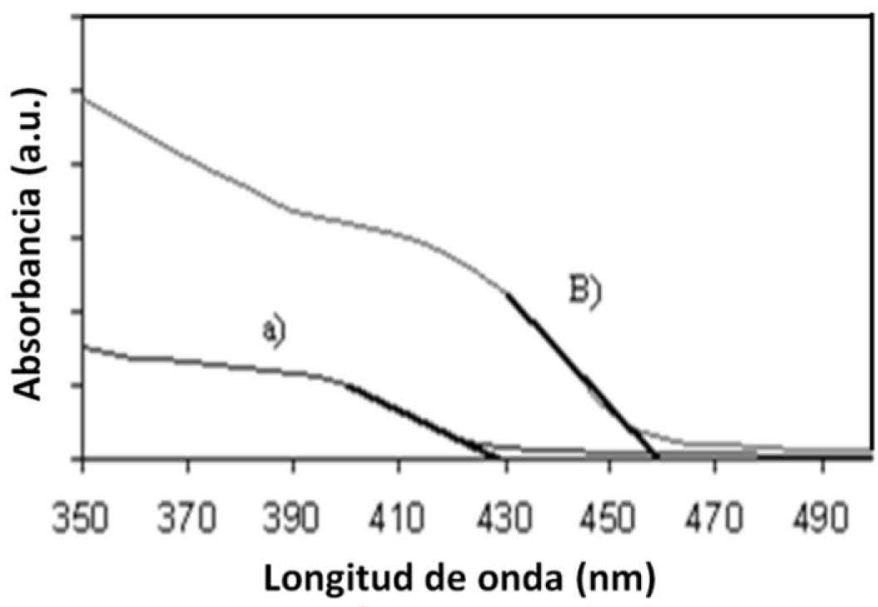

Figura 1. Espectro UV-Vis

Con la disminución del tamaño de partícula, la energía de banda prohibida del semiconductor se hace mayor. A esto se le llama efecto del tamaño quántico de la partícula del semiconductor ${ }^{4}$. A partir de los espectros de absorción de UV-Vis, la $\mathrm{E}_{\mathrm{g}}$ fue calculada utilizando la ecuación de Planck ${ }^{6}$ (Ec. 1) y presenta valores de $2.75 \mathrm{eV}$ y $2.95 \mathrm{eV}$ dependiendo del tamaño observado.

$$
E_{g}=h v=\frac{h c}{\lambda}=\frac{1240}{\lambda}
$$

Donde $\mathrm{h}$ es la constante de Planck, $\mathrm{u}$ es la frecuencia de la radiación electromagnética, c es la velocidad de la luz y $\lambda$ es la longitud de onda de absorción del semiconductor.

El análisis de DRX permitió confirmar la obtención de CdS, ya que las reflexiones obtenidas concuerdan con las reportadas para el semiconductor [10]. De acuerdo con los índices de Miller reportados para el material, se encontró que presenta una estructura hexagonal (wurzita). Otro aspecto importante en el difractograma es la obtención de señales anchas y números de cuentas bajos, lo cual se traduce con partículas de tamaño muy pequeño. De acuerdo con la ecuación de Sherrer (Ec. 2), el tamaño de las partículas que fueron precipitadas y analizadas por DRX tiene valores de alrededor de los $15 \mathrm{~nm}$.

$$
D=\frac{G \lambda}{B \cos \theta}
$$

Donde $\mathrm{D}$ es el diámetro de las partículas, $\lambda$ es la longitud de onda de la radiación a la cuál fue efectuado el análisis, $G$ es un parámetro que toma valor de 0.91 para CdS en sistema hexagonal, $B$ es la anchura a la mitad del pico de difracción y $\theta$ es el ángulo de difracción.

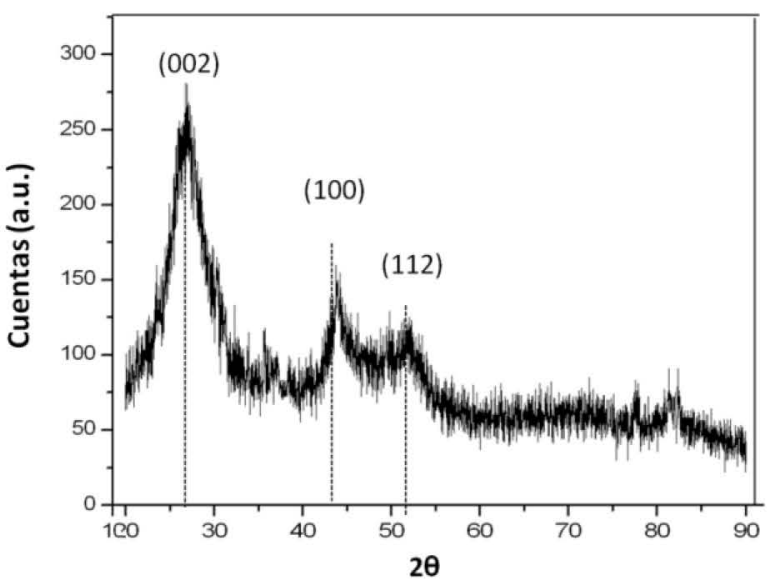

Figura 2. Difractograma de CdS sintetizado a partir de $\mathrm{CdCl} 2$ y TAA a una potencia del $80 \%$ 
Las muestras obtenidas de estos experimentos se analizaron por medio de las técnicas de UV-Vis, y de acuerdo a lo establecido por Wang, se calcularon los valores de tamaño de las partículas del sistema. Los resultados obtenidos permiten construir los gráficos de cinéticas de crecimiento. La figura 3, muestra el gráfico de crecimiento modelo que se encontró para las dispersiones estudiadas, en ella se puede observar la un crecimiento con tres pendientes, las cuales obedecen a distintos mecanismos de crecimiento. Las ecuaciones cinéticas de crecimiento siguen el siguiente modelo:

$$
(1-n)(\ln \alpha-\ln r)-\ln (n-1)=\ln K+\text { int }
$$

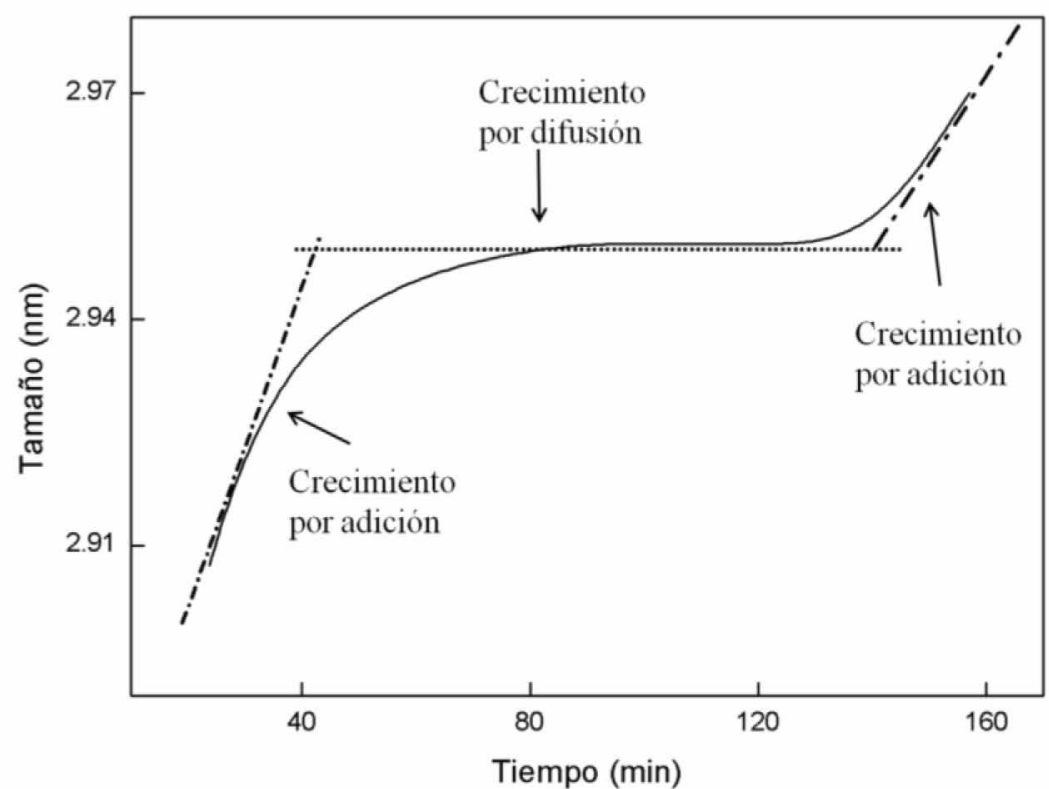

Figura 3. Modelo de los gráficos de crecimiento de las nanopartículas estudiadas en dispersión acuosa.

Donde $r$ es el radio de las partículas estudiadas, $t$ es el tiempo de crecimiento, $n$ es el orden de reacción y $\mathrm{K}$ es la constante cinética para el proceso. A partir de los resultados obtenidos con este modelo, se ajustó la ecuación 3 y se simplificó para la obtención de una ecuación de la forma:

$$
A r^{2}(\ln r-1)=B t+\ln k
$$

De acuerdo con los datos experimentales y el tratamiento matemático de dicha ecuación, la tabla 1 presenta los resultados obtenidos para cada uno de los parámetros de la ecuación, para los experimentos a diferentes concentraciones.

Tabla 2. Valores para A, B, K en la ecuación 2 para cada segmento de las etapas de crecimiento

\begin{tabular}{|c|c|c|c|c|c|c|c|c|c|}
\hline $\begin{array}{c}\text { Concentración } \\
\text { Citrato de } \\
\text { Sodio (mM) }\end{array}$ & \multicolumn{3}{|c|}{ Primer segmento } & \multicolumn{3}{c|}{ Segundo Segmento } & \multicolumn{3}{c|}{ Tercer segmento } \\
\hline & $\mathrm{A}$ & $\mathrm{B}$ & $\mathrm{K}$ & $\mathrm{A}$ & $\mathrm{B}$ & $\mathrm{K}$ & $\mathrm{A}$ & $\mathrm{B}$ & $\mathrm{K}$ \\
\hline 1.7 & 0.673 & 0.012 & 1.276 & 2.385 & 0.004 & 3.762 & 0.181 & 0.433 & 1.609 \\
\hline 0.8 & 2.100 & 0.019 & 2.947 & 0.200 & 0.003 & 1.127 & 3.400 & 1.020 & 9.025 \\
\hline 0.37 & 2.300 & 0.065 & 3.935 & 2.000 & 0.160 & 4.179 & --- & --- & -- \\
\hline
\end{tabular}

En este estudio se pudo comprobar que la presencia de citrato de sodio es de vital importancia para la estabilidad de las partículas, además se observó que la concentración del estabilizante tiene un papel importante ya que determina la cinética de crecimiento de las partículas presentes en el sistema. Como se puede observar en los resultados obtenidos, la concentración máxima de citrato de sodio utilizada, 1.7 $\mathrm{mM}$, presenta una estabilidad superior a las demás concentraciones utilizadas, e inclusive, la concentración menor, $0.37 \mathrm{mM}$, presenta una estabilidad mínima que propicia el aumento de tamaño de las partículas por mecanismos de adición. 
Además, tal como se mencionó anteriormente, el crecimiento se da en etapas, en las que primeramente se observa un crecimiento por mecanismos de adición con aumento en tamaño importante para las partículas a lo largo del tiempo. En segunda etapa se presenta una etapa de estabilidad, en la cual se observa un aumento de tamaño mínimo en las partículas hasta alcanzar un nuevo radio crítico, en este periodo las partículas de menor tamaño difunden a los huecos presentes en el aglomerado. Finalmente, en la tercera etapa se iniciar nuevamente el crecimiento por mecanismos de adición.

Posteriormente se realizó la deposición de las partículas sobre sustratos metálicos. Para este propósito se utilizaron dos tipos de acero los cuales se describen en la tabla 1. La Figura 4, muestra el CdS depositado sobre el sustrato de acero al manganeso, en el que se observó que el semiconductor forma pequeños aglomerados de partículas los cuales alcanzan tamaños de hasta $60 \mu \mathrm{m}$ aproximadamente. Además se presentaron estructuras con morfologías tipo agujas las cuales tienen un diámetro promedio de $3 \mu \mathrm{m}$; sin embargo, se pueden observar crecimientos similares con dimensiones inferiores. Tanto los aglomerados como las agujas se presentan de forma puntual dentro de la superficie del sustrato metálico, por lo que solo sitios específicos fueron susceptibles al crecimiento del semiconductor.
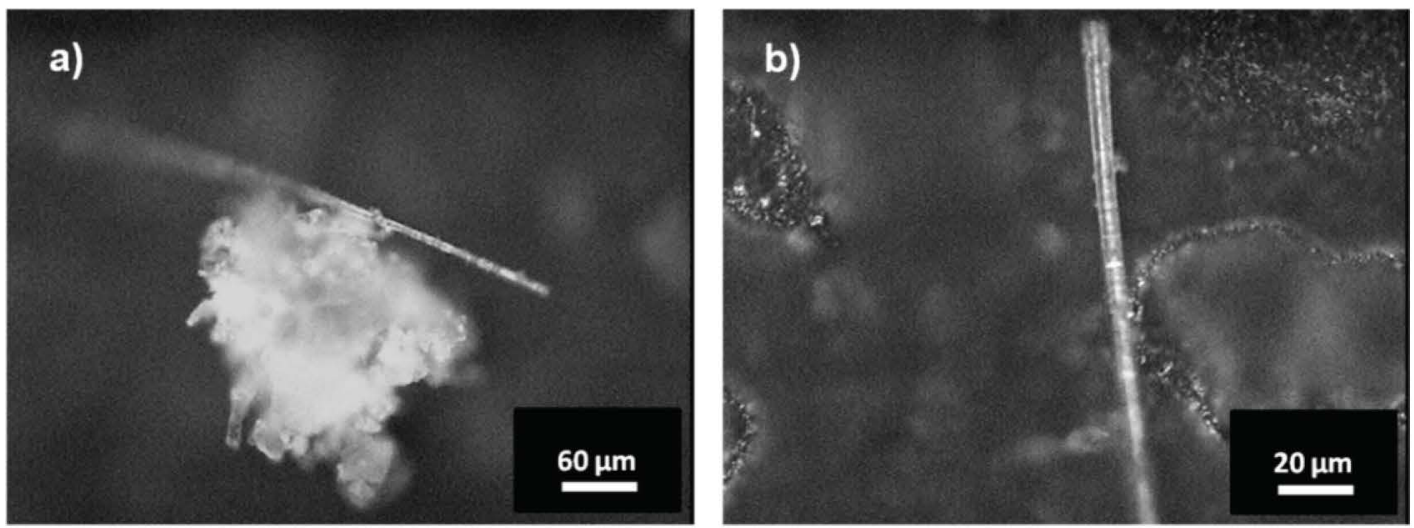

Figura 4. Micrografías de CdS depositado en el sustrato metálico que contiene Mn $0.25 \%$, C $0.10 \%$ y Fe $99.65 \%$.

Se continuó con la deposición sobre el segundo sustrato metálico que consiste de un acero al silicio. La Figura 5 micrografías para dicho experimento, obsérvese que en este sustrato el CdS forma una capa uniforme sobre toda la superficie lo cual indica que la afinidad del CdS por este tipo de acero es mucho mayor que en el caso anterior. Este fenómeno nos lleva a la formación de películas delgadas del semiconductor sobre el sustrato metálico las cuales presentaron buena estabilidad.
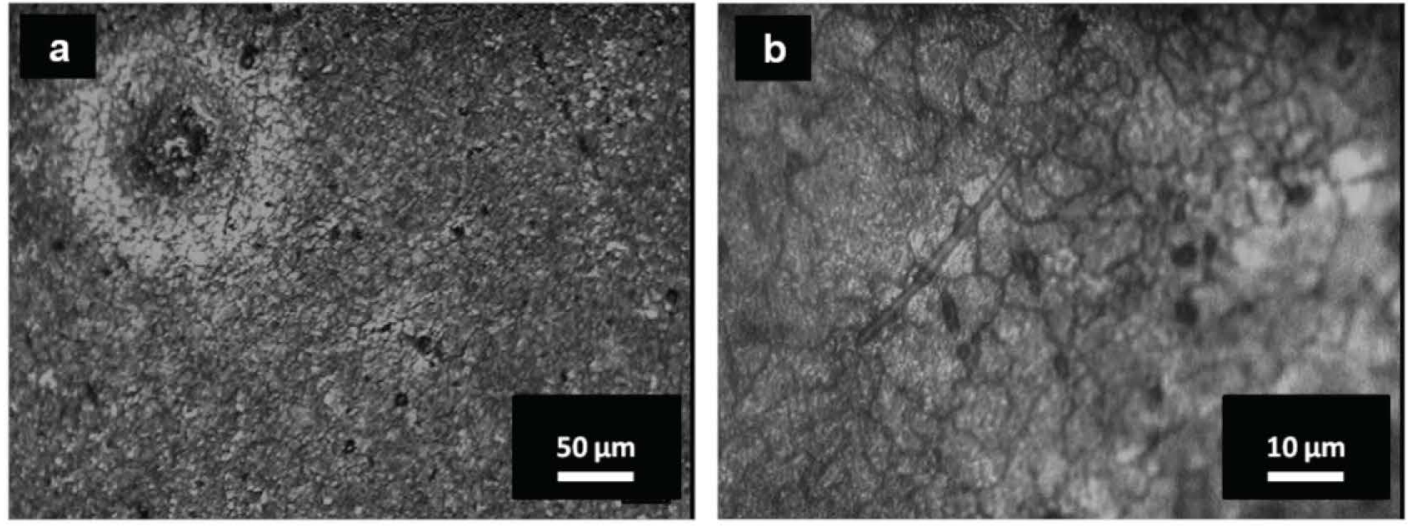

Figura 5. Micrografías de CdS depositado en el sustrato metálico que contiene Si $0.45 \%$, C $0.01 \%$ y Fe $99.51 \%$. 


\section{Conclusiones}

A partir de los datos cinéticos se observó que las partículas siguen un crecimiento en tres etapas, lo que habla de diferentes modelos de crecimiento, adición y difusión. Además se observó que existe una variación directamente proporcional entre la concentración de citrato de sodio y la estabilidad del sistema, ya que al disminuir la concentración del estabilizador los tamaños de las partículas se incrementan y la estabilidad de la dispersión coloidal se ve comprometida. A partir de los tratamientos térmicos se observó la afinidad del semiconductor por aquel sustrato que contenía silicio, ya que se observó la formación de una película uniforme. Para el sustrato que contenía Mn se observó que el sistema sólo se deposita en ciertos sitios activos y que el crecimiento se da sobre las partículas anteriormente depositadas.

\section{Referencias}

[1] R. Trotta, L. Cavigli, L. Felisari, A. Polimeni, A. Vinattieri, M. Gurioli, M. Capizzi, F. Martelli, S.

Rubini, L. Mariucci, M. Francardi, A. Gerardino. Phys. Rev. B 2010, 81, 235327-235331.

[2] W. Wu, H. Ye, X. Ruan. Nanotechnology 2010, 2126570

[3]A.E Raevskaya, A.L Stroyuk, S.Y Kuchmiy. J Colloid Interface Sci. 2006, 302, 1, 133-41.

[4] W. Xu and D. L. Akins. Materials Letters . 2004, 58, 21, 2623-2626

[5] V. Popescu, H. I. Naşcu, E. Darvasi. Journal of Optoelectronics and Advanced Materials, 2006, $8,3,1187-1193$

[6] Y. Wang, N. Herron . J. Phys. Chem 1991, 95, 525-532

[7] S. Martínez, T. Serrano, I. Gómez, A. Hernández. Bol. Soc. Esp. Ceram. V. 2007, 46 [2] 97-101

[8] S. Kar, ${ }^{\ddagger}$ B. Satpati, ${ }^{ \pm}$P. V. Satyam, ${ }^{ \pm}$and S. Chaudhuri. J. Phys. Chem. B, 2005, 109 (41), pp 19134-19138

[9] P. Zhang, C. Cheng, P. Jiao, Y. Li. Materials Letters, 2008, 62, 8-9, 31, 1151-1154

[10] B. Liu, J.Y. Lee. The Journal Of Physical Chemistry B Letters 2005,109,23783-23784. 